\title{
Boas Práticas de Laboratório: COMO SURGIRAM? O QUE SÃO? A QUE SE APLICAM?
}

\author{
Cristina Galacho*
}

\begin{abstract}
Nesterir este artigo apresenta-se uma panorâmica geral sobre Boas Práticas de Laboratório (BPL) e os Princípios da OCDE de BPL, nomeadamente evolução histórica, definição, âmbito de aplicação, principais objetivos e legislação. Aborda-se o enquadramento legal das BPL em Portugal, as autoridades nacionais competentes para o seu acompanhamento e os trâmites do processo de certificação de acordo com os Princípios da OCDE de BPL. Referem-se ainda as Entidades Nacionais com certificação de conformidade na área dos Medicamentos e Produtos Cosméticos, e na área dos Produtos Químicos Industriais.
\end{abstract}

\section{HISTORIAL}

As Boas Práticas de Laboratório (BPL) surgiram pela primeira vez em texto legislativo, na Nova Zelândia, em 1972, e na Dinamarca, em 1973, com o objetivo de credibilizar a prática laboratorial no domínio da investigação e desenvolvimento [1-3].

Em 1976 surgiu a primeira proposta "formal" de regras de BPL (Good Laboratory Practice Regulations: Proposed Rule), como resposta a práticas incorretas verificadas pela agência norte americana FDA (do inglês US Food and Drug Administration) em atividades de investigação e desenvolvimento realizadas em laboratórios de companhias farmacêuticas e/ou laboratórios contratados pelas mesmas, nomeadamente, planeamento inadequado e execução incompetente de estudos, documentação insuficiente relativa a métodos e resultados e, em alguns casos, fraude.

Três anos mais tarde, em 1979, entrou em vigor a proposta final da FDA (Good Laboratory Practice Regulations: Final Rule 21 CFR 58), a primeira legislação com verdadeiro impacto internacional, tendo sido estabelecidas regras de BPL para que nos estudos de conceção e ensaio de um produto, no contexto do registo de novos fármacos, os resultados ob-

* Departamento de Química, Escola de Ciências e Tecnologia, Universidade de Évora

Centro de Química de Évora

E-mail: pcg@uevora.pt tidos fossem cientificamente válidos [1-6].

No domínio da Química, nomeadamente na área dos pesticidas, a agência de proteção ambiental norte americana EPA (do inglês US Environmental Protection Agency) deparou-se com problemas similares, publicando em 1983 as regras finais de BPL (40 CFR 160 e 40 CFR 792) [2, 4].

Um dos casos mais famosos e ilustrativo de falhas no domínio das práticas laboratoriais data de 1976, apesar de só ter sido notícia nos meios de comunicação social em 1983, e diz respeito aos Laboratórios Industriais Bio-Test, IBT (do inglês Industrial Bio-Test Laboratories). Os IBT, sediados em Illinois, eram, à data, responsáveis por mais de 20000 estudos de segurança e eficácia de centenas de pesticidas e outros produtos químicos, o que correspondia a $35-40 \%$ do número total de estudos de toxicologia realizados nos Estados Unidos da América. As alegadas irregularidades cometidas por estes laboratórios incluíram a falsificação de dados, a reposição de animais que pereceram durante 0 estudo por animais novos (os quais não tinham sido tratados de forma apropriada com o composto teste) e sem documentação da substituição, produção de resultados e exclusão de resultados quando os mesmos poderiam não ser considerados favoráveis pelas autoridades oficiais. Estes laboratórios foram considerados culpados e os responsáveis foram devidamente punidos por defraudar o governo ame- ricano em consequência da falsificação de resultados que serviram como suporte ao registo de pesticidas, cosméticos, produtos farmacêuticos de uso humano e animal, e de outras substâncias químicas [2, 4].

De entre as práticas incorretas realizadas constavam os estudos referentes ao glifosato ${ }^{1}$, solicitados pela multinacional Monsanto, no sentido de satisfazer as exigências de registo. O IBT realizou cerca de 30 testes com glifosato e produtos à base de glisofato, inclusive 11 dos 19 estudos sobre toxicologia crónica. Um exemplo concreto da péssima qualidade dos dados do IBT está bem patente na declaração de um perito da EPA: "É difícil não duvidar da integridade científica de um estudo do IBT quando neste se afirma que foram retiradas amostras do útero (de coelhos machos) para exames histomorfológicos" [7].

As anomalias detetadas pela FDA e EPA, pondo em evidência o eventual risco para a saúde pela aprovação de novas substâncias destinadas a produtos farmacêuticos, pesticidas e outros produtos químicos com base em estudos incompletos, deficientes ou mesmo fraudulentos, foram indubitavelmente determinantes para a regulamentação e implementação das Boas Práticas de Laboratório.

A nível internacional, a Organização para a Cooperação e o Desenvolvimento Económico, OCDE, enunciou pela primeira vez em 1981 os Princípios de Boas Práticas de Laboratório 
como resultado do trabalho de um grupo internacional de peritos, desenvolvido entre 1979 e 1980, no âmbito de um programa sobre o controlo de produtos químicos [C(81)30(Final)]. Após 15 anos de aplicação com sucesso, devido ao progresso científico e técnico no campo dos testes de segurança e ao facto dos mesmos serem requeridos em muito mais áreas do que no final da década de 70 do século passado, os Princípios da OCDE de BPL foram revistos e atualizados, entre 1995 e 1996, por um novo grupo internacional de peritos (incluindo portugueses), e adotados no conselho da OCDE em 1997 [C(97)186(Final)] [3, $4,8,9]$.

A OCDE tem editados 15 documentos $^{2}$ relativos aos Princípios de BPL e verificação da conformidade e, ainda, 2 artigos de posição [9].

Em termos de legislação Europeia, a então CEE adotou, pela $1 .^{\mathrm{a}}$ vez, em 1986, a Diretiva do Conselho n. ${ }^{\circ}$ 87/18/CEE, de 18 de dezembro, relativa à aproximação das disposições legislativas, regulamentares e administrativas respeitantes à aplicação dos Princípios BPL e ao controlo da sua aplicação para os ensaios sobre as substâncias químicas [10].

Em 1988 foi adotada a Diretiva do Conselho n. ${ }^{\circ}$ 88/320/CEE, de 7 de junho, relativa à inspeção e verificação das BPL [11]. Posteriormente as mesmas foram revistas e alteradas, com base nos Princípios da OCDE de BPL (revisão de 1997), estando vigentes, respetivamente, as Diretivas 2004/10/ CE e 2004/09/CE do parlamento Europeu e do Conselho, ambas de 11 de fevereiro de 2004 (versões codificadas) $[8,12]$.

BPL E PRINCÍPIOS DA OCDE DE BPL: DEFINIÇÃO, ÂMBITO DE APLICAÇÃO E OBJETIVOS

Por BPL entende-se um sistema de qualidade relacionado com o processo de organização e as condições segundo as quais são planeados, executados, acompanhados, registados, arquivados e apresentados os resultados de estudos não clínicos ${ }^{3}$ de segurança para a saúde e ambiente $[3,8,9]$.
Por sua vez, o termo "Princípios BPL" designa as BPL conformes com os Princípios da OCDE de BPL $[12,13]$. Como tal, os Princípios da OCDE de BPL são constituídos por um conjunto de critérios normativos destinados ao estabelecimento do sistema de qualidade anteriormente referido e incluem os seguintes aspetos fundamentais:

1) Organização e pessoal da instalação de ensaio.

2) Programa de garantia da qualidade.

3) Instalações.

4) Equipamentos, materiais e reagentes.

5) Sistemas de ensaio.

6) Substâncias para estudo e de referência.

7) Procedimentos habituais de funcionamento.

8) Execução do estudo.

9) Elaboração de relatórios do estudo.

10) Armazenamento e conservação de registos e materiais.

O âmbito de aplicação dos Princípios da OCDE de BPL abrange os ensaios de segurança não clínicos de substâncias em estudo que entrem na composição de produtos farmacêuticos, pesticidas, cosméticos, medicamentos veterinários, bem como de aditivos alimentares, aditivos para alimentos de animais e produtos químicos industriais, visando a obtenção de dados, ainda não conhecidos, sobre as suas propriedades e/ou sobre a sua segurança para a saúde humana ou animal e/ou o ambiente. Estas substâncias são frequentemente produtos químicos sintéticos, mas podem também ser de origem natural ou biológica e, em algumas circunstâncias, organismos vivos.

Os estudos não clínicos de segurança para a saúde e o ambiente incluem trabalhos realizados em laboratórios, estufas e no terreno.

Exceto quando especificamente isentos pela legislação nacional, os Princípios da OCDE de BPL aplicam-se a todos os ensaios não clínicos de segurança toxicológica, com reflexos na saúde humana ou animal e na qualidade ambiental, regulamentarmente exigidos para fins de concessão de licenças ou para registo dos produtos anteriormente referidos, bem como para regulamentação de produtos químicos industriais $[3,8]$.

Em última instância, o objetivo dos Princípios da OCDE de BPL é promover o desenvolvimento de dados e resultados de ensaio com qualidade. A possibilidade de comparação dos dados dos ensaios constitui a base para a sua aceitação mútua entre os países membros da OCDE. Se cada país puder confiar nos dados de ensaios desenvolvidos noutros países, é então possível evitar a duplicação de ensaios, poupando-se assim tempo e recursos. Adicionalmente, o reconhecimento mútuo dos resultados dos ensaios obtidos por utilização de métodos normalizados e reconhecidos é condição essencial para a redução do número de experiências executadas em animais, tal como disposto na Diretiva do Conselho n. ${ }^{\circ}$ 86/609/EEC de 24 de novembro de 1986.

A aplicação destes princípios deve contribuir para evitar a criação de barreiras técnicas ao comércio e aumentar o nível de proteção da saúde humana e do ambiente [3, 8, 12].

\section{Princípios da OCDE de BPL em Portugal}

Em Portugal, o Decreto-Lei n. ${ }^{\circ}$ 95/2000, de 23 de maio, transpõe para o ordenamento jurídico interno a Diretiva n. ${ }^{\circ}$ 99/12/CE e estabelece as regras relativas à inspeção e verificação dos Princípios da OCDE de BPL. Por sua vez, o Decreto-Lei n. ${ }^{\circ}$ 99/2000, de 30 de maio, transpõe para o ordenamento jurídico interno a Diretiva n. ${ }^{\circ}$ 87/18/CEE, relativa à aplicação dos Princípios da OCDE de BPL e ao controlo da sua aplicação para os ensaios sobre as substâncias químicas, e a Diretiva n. ${ }^{\circ}$ 99/11/CE, que adapta ao progresso técnico os princípios contidos naquela diretiva $[3,13]$.

Estes Decretos-Lei, DL n. ${ }^{\circ}$ 95/2000 e n. ${ }^{\circ}$ 99/2000, constituem a legislação nacional atualmente em vigor, 
fazem referência direta aos Princípios da OCDE de BPL (revisão de 1997) e correspondem às disposições contidas nas diretivas 2004/9/CE e 2004/10/CE (versões codificadas).

As autoridades nacionais de acompanhamento competentes para avaliação e verificação da conformidade com os Princípios da OCDE de BPL são a Autoridade Nacional do Medicamento e Produtos de Saúde I.P., INFARMED, e o Instituto Português da Qualidade I.P., IPQ, conforme o disposto nos artigos 3. ${ }^{\circ}$ do Decreto-Lei n. ${ }^{\circ} 95 / 2000$ e $6 .^{\circ}$ do Decreto-Lei n. ${ }^{\circ} 99 / 2000[3,13,14,16,17]$.

O INFARMED, Instituto Público integrado no Ministério da Saúde, foi criado em 1993 e é autoridade com atribuições nos domínios da avaliação, autorização, disciplina, inspeção e controlo de produção, distribuição, comercialização e utilização de medicamentos de uso humano, incluindo os medicamentos à base de plantas e homeopáticos, e de produtos de saúde (que incluem produtos cosméticos e de higiene corporal, dispositivos médicos e dispositivos médicos para diagnóstico in vitro) [15], estando-lhe atribuída a responsabilidade pelos assuntos respeitantes ao sistema BPL relativos a esta área [3, 13, 14].

Por sua vez o IPQ, Instituto Público integrado no Ministério da Economia e do Emprego, foi criado em 1985, e tem por missão, nos termos da sua lei orgânica aprovada pelo Decreto-Lei n. ${ }^{\circ} 71 / 2012$ de 21 de março, a coordenação do Sistema Português da Qualidade, SPQ, e de outros sistemas de qualificação regulamentar que lhe forem conferidos por lei, a promoção e a coordenação de atividades que visem contribuir para demonstrar a credibilidade da ação dos agentes económicos, bem como o desenvolvimento das atividades inerentes à sua função de laboratório nacional de metrologia $[16,18]$.

O Sistema Português da Qualidade congrega os subsistemas da normalização, da qualificação e da metrologia, sendo o IPQ o Organismo Nacional da Normalização e a Instituição Nacional de Metrologia [18]. No âm- bito do subsistema de metrologia foi-Ihe atribuída a responsabilidade pelo acompanhamento do sistema de BPL na área dos outros produtos químicos e produtos para a proteção das plantas $[3,13,14,16-18]$.

O sistema de reconhecimento de laboratórios segundo os Princípios OCDE de BPL, gerido pelo INFARMED e pelo IPQ, em conformidade com o DL n. ${ }^{\circ}$ 95/2000, de 23 de maio, segue os critérios estabelecidos na Diretiva 2004/9/CE.

A certificação de conformidade com as BPL é concedida a qualquer instalação de ensaio (laboratório) nacional, público ou privado, que pretenda declarar e aplicar as BPL e evidencie a conformidade com os Princípios da OCDE de BPL, tal como estabelecido no DL n. ${ }^{\circ}$ 99/2000, de 30 de maio, realizando-se a avaliação tal como referido no anexo ao DL n. ${ }^{\circ}$ 95/2000, de 23 de maio [14].

Os critérios gerais são complementados pelo conjunto de documentos orientadores editados pela OCDE e anteriormente referidos.

A tramitação do processo de certificação BPL decorre de acordo com o estipulado no Procedimento de Candidatura e no Programa Nacional de Acompanhamento BPL, programa nacional de verificação do cumprimento das BPL que assegura que as instalações de ensaio implementaram adequadamente os Princípios BPL para a execução dos estudos e estão em condições de garantir a qualidade adequada dos dados resultantes dos mesmos [17], cujos documentos se encontram disponíveis na página web do INFARMED [14].

Caso seja conferido o reconhecimento com os Princípios da OCDE de BPL é emitido, pela autoridade competente e ao abrigo do DL 95/2000 de 23 de maio, um certificado de conformidade válido por um período de dois anos renovável.

Atendendo ao facto do Programa Nacional de Acompanhamento de BPL estar reconhecido pela UE e pela OCDE, os dados gerados por instalações de ensaio do referido programa serão aceites pela maioria das Autoridades Regulamentares de outros países, baseadas na decisão da OCDE de 12 de maio de 1981 de aceitação mútua de dados "OCDE MAD" [17].

Atualmente existem em Portugal cinco laboratórios com certificação de conformidade com os Princípios da OCDE de BPL, na área dos Medicamentos e Produtos Cosméticos, nomeadamente, o Laboratório de Estudos Farmacêuticos, LEF, o Labor Qualitas, a Unidade de Serviços Analíticos do Instituto de Biologia Experimental e Tecnológica e do Instituto de Tecnologia Química e Biológica, ASU do IBET/ITQB, o Centro de Estudos de Farmacologia - AIBILI e o Laboratório de Investigação Farmacológica BIAL [14]. A título exemplificativo refiram-se:

- O LEF, entidade prestadora de serviços ao sector farmacêutico, assim como a outras áreas, tais como a alimentar e a de produtos cosméticos e de higiene corporal, cujo departamento bioanalítico possui certificação BPL/OCDE, concedida pelo INFARMED e pela ANVISA (Agência Nacional de Vigilância Sanitária, Brasil) [19];

- O Labor Qualitas, pólo de investigação e desenvolvimento do grupo Tecnimede, GTM, que é certificado pelo INFARMED, pelo IPAC para a norma NP EN/IEC ISO 17025 e também para as Boas Práticas de Fabrico, sendo um dos laboratórios a nível europeu com a capacidade técnica para análise e libertação de medicamentos. De acordo com o GTM, o compromisso com a excelência permitiu a conclusão com sucesso de mais de 80 produtos farmacêuticos para uso humano, que geraram, até à data, mais de 2000 autorizações de introdução no mercado a nível mundial [20];

- A Unidade de Serviços Analíticos, ASU, do Instituto de Biologia Experimental e Tecnológica, IBET, e Instituto de Tecnologia Química e Biológica, ITQB, é certificada de acordo com os princípios OCDE de BPL pelo INFARMED. Refira-se que entre 2007 e 2010 esta unidade foi certificada simultaneamente pelo INFARMED e pelo IPQ [21]. 
No que respeita à área de Produtos Químicos Industriais, a SAPEC AGRO, S.A., é a única entidade reconhecida no Programa Nacional de Acompanhamento de BPL pelo IPQ $[16,22]$. A obtenção de dados experimentais no âmbito de sistemas de qualidade de acordo com as BPL, necessários ao processo de registo de produtos fitofarmacêuticos, constitui um dos pilares da política de gestão do grupo SAPEC [22]. No âmbito dos Princípios da OCDE de BPL, a SAPEC AGRO tem certificados o Laboratório da Divisão Industrial de Investigação e Desenvolvimento (Laboratório Físico/Químico), a Unidade de Ensaios de Resíduos e o Laboratório de Resíduos [16, 22].

A título exemplificativo apresenta-se, na Figura 1, o Certificado de Conformidade do Laboratório BPL da Divisão Industrial de Investigação e Desenvolvimento da SAPEC AGRO, S.A., emitido pelo IPQ.

\section{Outras Boas Práticas}

Para além das BPL (GLP, do inglês Good Laboratory Practice), que têm como missão basilar aumentar o nível de proteção da saúde humana e do ambiente, existe uma grande panóplia de outras Boas Práticas como, por exemplo, Boas Práticas de Fabrico (GMP, do inglês Good Manufacturing Practice), Boas Práticas de Distribuição (GDP, do inglês Good Distribution Practice) e Boas Práticas Clínicas (GCP, do inglês Good Clinical Practice).

\section{Agradecimentos}

A autora agradece à Dr. ${ }^{\text {a }}$ Cristina Silva Oliveira do Grupo BPL - IPQ, à Dr. $^{\text {a }}$ Mónica Galo do INFARMED, à Dr. ${ }^{a}$ Isabel Murta do ITQB-UNL e ao Eng. ${ }^{\circ}$ Pedro Vilas Boas da SAPEC AGRO, S.A., pela documentação enviada e esclarecimentos prestados, e ao(à) revisor(a) pelas sugestões/ críticas efetuadas.

\section{NOTAS}

1 O glifosato ( $\mathrm{N}$-(fosfonometil)glicina, $\mathrm{C}_{3} \mathrm{H}_{8} \mathrm{NO}_{5} \mathrm{P}$ ) é um herbicida pós-emergente classificado como não-seletivo e de ação sistémica. Apresenta largo espectro de ação, o que possibilita o controlo de plantas daninhas anuais ou perenes [23]

Em 1970, o glifosato foi patenteado pela Monsanto e comercializado sob marca Roundup ${ }^{\circledR}$. A partir de 2000, data em que expirou a referida patente, começou a ser produzido e vendido sob diferentes marcas registadas sendo, atualmente, um dos mais usados nos Estados Unidos da América.

Existe alguma controvérsia quanto aos possíveis efeitos tóxicos dos herbicidas que contêm glifosato, em termos de segurança ambiental e saúde humana, embora os mesmos sejam classificados, pela EPA, como levemente tóxicos (classe III) [23, 24].

2 Documentos da OCDE relativos aos Princípios de BPL e Verificação da Conformidade [9]:

\section{Princípios da OCDE de BPL:}

N. ${ }^{\circ}$ 1: Princípios da OCDE de BPL.

\section{Documentos de Consenso:}

N. ${ }^{\circ}$ 4: Garantia da Qualidade e BPL;

N. ${ }^{\circ}$ 5: Conformidade dos fornecedores de laboratórios com os Princípios de BPL;

N. ${ }^{\circ}$ 6: Aplicação dos Princípios de BPL a estudos de campo;

N. ${ }^{7}$ : Aplicação dos Princípios de BPL a estudos de curta duração;

N. ${ }^{\circ}$ 8: Funções e responsabilidades do diretor do estudo de BPL;

N. ${ }^{\circ}$ 10: Aplicação dos Princípios de BPL a sistemas informatizados;

N. ${ }^{\circ}$ 13: Aplicação dos Princípios de BPL à organização e realização de estudos plurilocais.

Documentos de Orientação para as Autoridades de Verificação da Conformidade:

N. ${ }^{\circ}$ 2: Guia revisto dos procedimentos de verificação da conformidade de BPL;

N. ${ }^{\circ}$ 3: Guia revisto para a inspeção de laboratórios e auditoria dos estudos;

N. 9: Guia para a preparação de relatórios de inspeção de BPL.

Documentos de Aconselhamento do Grupo de Trabalho BPL:

N. ${ }^{\circ}$ 11: Funções e responsabilidades do patrocinador na aplicação dos Princípios de BPL;

N. ${ }^{\circ}$ 12: Recomendações relativas ao pedido e realização de inspeção e de verificação dos estudos noutro país;

N. ${ }^{\circ}$ 14: Aplicação dos Princípios de BPL a estudos in vitro;

N. ${ }^{\circ}$ 15: Estabelecimento e controlo de arquivos que funcionam em conformidade com os Princípios de BPL.
3 Por estudo não clínico de segurança para a saúde e ambiente entende-se uma experiência ou um conjunto de experiências em que uma substância em estudo é analisada, em condições laboratoriais ou em ambiente normal, a fim de se obterem dados sobre as suas propriedades e/ou segurança, com vista à sua apresentação às autoridades regulamentares competentes $[3,8,9]$.

\section{REFERÊNCIAS}

[1] M. A. Anderson, GPL Quality Audit Manual, $3^{\text {rd }}$ Ed. Interpharm/CRC Press, Florida, 2000.

[2] J. P. Seiler, Good Laboratory Practice - the Why and the How, Springer-Verlag, Berlin Heidelberg, 2005.

[3] Decreto-Lei n. ${ }^{9}$ 99/2000 de 30 de maio, Diário da República - I Série-A, N. ${ }^{\circ} 125,30$ de maio de 2000.

[4] UNDP/World Bank/WHO Special Programme for Research and Training in Tropical Diseases (TDR), Handbook Good Laboratory Practice (GLP), 2000.

[5] J. C. Marques, http://www3.uma.pt/jcmarques/gmpglp.htm (J. C. Marques GMP/GLP, acedido em 15-03-2012).

[6] M. G. Slomiany, The Indispensable Guide to Good Laboratory Practice $(G L P), 2^{\text {nd }} E d$., Pinehurst Press, Inc., New York, 2009.

[7] C. Cox, Journal of Pesticides Reform 18 (1998), http://www.mindfully. org/Pesticide/Roundup-GlyphosateFactsheet-Cox2.htm (Mindfully.org, acedido em 14-03-2012).

[8] Directiva 2004/10/CE do Parlamento Europeu e do Conselho de 11 de fevereiro de 2004, Jornal Oficial da União Europeia, L 50/44-59. PT. 20.2.2004

[9] OCDE - Good Laboratory Practice (GLP),

http://www.oecd.org/document/1/0,374 6,en_2649_37465_48477249_1_1_1_ 37465,00.html

(OCDE - Good Laboratory Practice (GLP), acedido em 14-03-2012).

[10] Directiva 87/18/CEE do Conselho de 18 de dezembro de 1986, Jornal Oficial das Comunidades Europeias, N. ${ }^{\circ}$ L 15/29-30, 17.1.87.

[11] Directiva 88/320/CEE do Conselho de 9 de junho de 1988, Jornal Oficial das Comunidades Europeias, N. ${ }^{\circ} \mathrm{L}$ 145/35-37, 11.6.88.

[12] Diretiva 2004/9/CE do Parlamento Europeu e do Conselho de 11 de feve- 
reiro de 2004, Jornal Oficial da União Europeia, L 50/28-42. PT. 20.2.2004.

[13] Decreto-Lei n. ${ }^{9} 95 / 2000$ de 23 de maio, Diário da República - I Série-A, N. ${ }^{\circ} 119,23$ de maio de 2000.

[14] INFARMED - BPL, http://www. infarmed.pt/portal/page/portal/INFARMED/MONITORIZACAO_DO_ MERCADO/INSPECCAO/LABORATORIO_CONTROLO_QUALIDADE (Autoridade Nacional do Medicamento e Produtos de Saúde I.P., acedido em 12-07-2012).

[15] INFARMED - Sobre o INFARMED, http://www.infarmed.pt/portal/page/ portal/INFARMED/PERGUNTAS_ FREQUENTES/SOBRE_INFARMED (Autoridade Nacional do Medicamento e Produtos de Saúde I.P., acedido em 12-07-2012).

[16] IPQ, http://www.ipq.pt/backhtmlfiles/ ipq_mei.htm (Instituto Português da Qualidade, acedido em 12-07-2012).

[17] Decreto-Lei n. ${ }^{0} 71 / 2012$ de 21 de março, Diário da República - I Série, N. ${ }^{\circ}$ 58, 21 de março de 2012.

[18] Programa Nacional de Acompanhamento de Boas Práticas de Laboratório. Instituto Português da Qualidade. Departamento de Metrologia. Fevereiro de 2012.

[19] LEF, http://www.lef.pt/00Home. aspx?lg=pt (Laboratório de Estudos Farmacêuticos, acedido em 12-03-2012).

[20] Labor Qualitas - Grupo TECNIMEDE, http://www.tecnimede.com/index. php?cat=31\&id_empresa=47 (Labor Qualitas - Grupo TECNIMEDE, acedido em 12-03-2012).

[21] ITQB, http://www.itqb.unl.pt/facilities/ Analytical\%20Services/scientificservices/?searchterm=ASU (Instituto de Tecnologia Química e Biológica (UNL) - Analytical Services Unit, ace- dido em 12-07-2012).

[22] SAPEC AGRO, http://www.sapecagro.pt/internet/empresa (SAPEC AGRO, acedido em 28-06-2012).

[23] Monsanto, http://www.monsanto. com.br/produtos/herbicidas/roundup/ roundup.asp (Monsanto Herbicidas, acedido em 18-03-2012).

[24] Wikipedia glifosato, http:// es.wikipedia.org/wiki/Glifosato\#cite_ note-EPAusage-0 (Wikipedia glifosato, acedido em 21-03-2012).

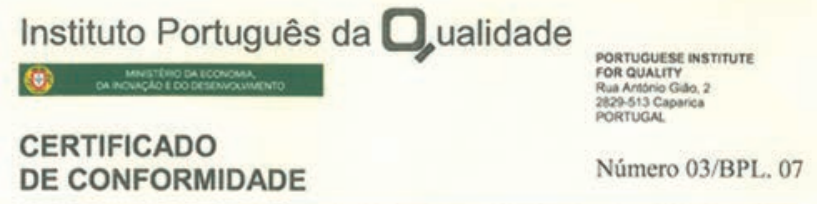

\section{O INSTITUTO PORTUGUÊS DA QUALIDADE certifica que o}

Laboratório BPL da Divisão Industrial de Investigação e Desenvolvimento da SAPEC Agro, S.A,

\begin{abstract}
Herdade das Praias
2901-852 Setúbal

É reconhecido para realizar ensaios físico-quimicos para caracterização analitica das substâncias activas e das preparações de produtos fitofarmacêuticos, em conformidade com os "Princípios da OCDE das Boas Práticas de Laboratório - BPL”, nos termos das Directivas 2004/9/CE e 2004/10/CE de 11 de Fevereiro, versão codificada.
\end{abstract}

O presente certificado, é emitido ao abrigo Decreto Lei $\mathrm{n}^{\circ}$ 95/2000 de 23 de Maio, é válido pelo periodo de dois anos renovável e substitui o anteriormente emitido em 18 de Julho de 2008.

Monte da Caparica, 12 Abril de 2010

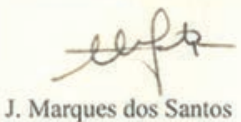

Presidente do Conselho Directivo

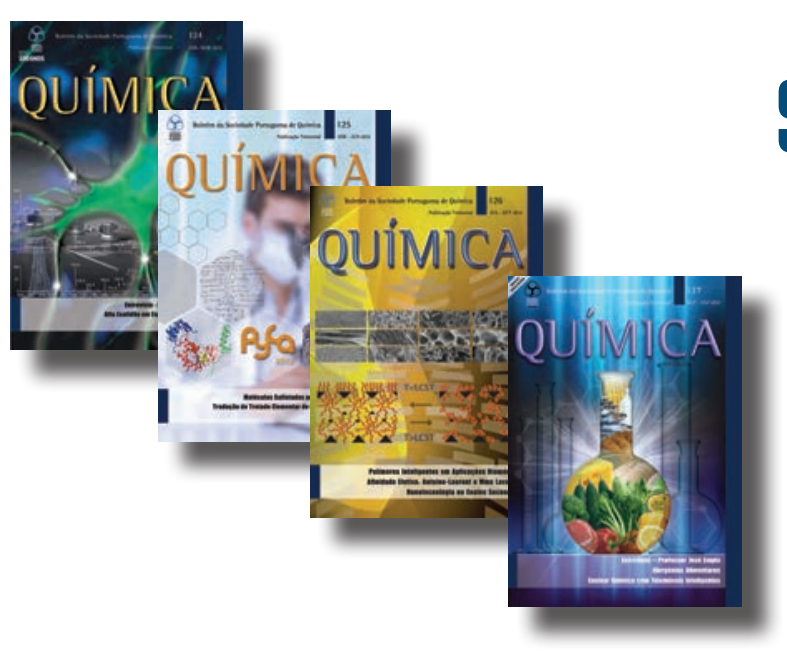

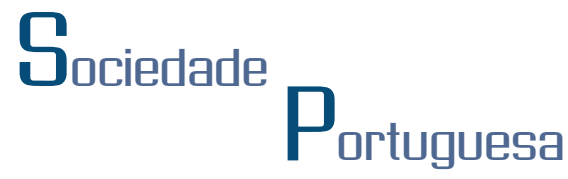

${ }^{\mathrm{de}} \square_{\text {uirinica }}$ 
